\title{
REMARKS ON A MATRIX TRANSFORMATION FOR LINEAR DIFFERENTIAL EQUATIONS
}

\author{
WILLIAM T. REID
}

1. Introduction. Consider the vector differential equation

$$
y^{\prime}=A(x) y,
$$

where $A(x) \equiv\left\|A_{i j}(x)\right\|$ is an $n \times n$ matrix with elements that are complex-valued continuous functions of the real variable $x$ on a given interval $\Delta$. For brevity a nonsingular $n \times n$ matrix $T(x)$ with continuously differentiable elements on $\Delta$ will be termed an admissible transformation matrix; for such a $T(x)$ the equation (1) is equivalent under the transformation $y=T u$ to the equation

$$
u^{\prime}=B(x) u, \text { where } B=T^{-1}\left(A T-T^{\prime}\right) .
$$

The remarks of this note are concerned with a result on transformations stated below as Theorem A, and are two-fold in nature: firstly, there are comments on the relation of this theorem to results of Perron [3] and Diliberto $[1 ; 2]$, in the hope of correcting a misunderstanding that has arisen in this regard; secondly, there are remarks stressing two general properties of admissible transformation matrices which together afford a very elementary matrix proof of Theorem A.

Matrix notation will be used throughout, with a vector considered as a one-column matrix. If $M$ is a matrix then the corresponding transpose and conjugate-transpose matrices are denoted by $\tilde{M}$ and $M^{*}$, respectively. The symbol $|y|$ will be employed for the norm $\left(y^{*} y\right)^{1 / 2}$ of a vector $y$. For $M \equiv\left\|M_{i j}\right\|,(i, j=1, \cdots, n)$, the corresponding lower case bold-face letter $\boldsymbol{m}_{j}$ will denote the $j$ th column vector of $M$. In particular, if $M$ is a nonsingular $n \times n$ matrix, and $N$ is the unitary matrix whose sequence of column vectors $n_{1}, \cdots, n_{n}$ is the set of vectors obtained by applying the Gram-Schmidt orthonormalization process to the sequence of column vectors $m_{1}, \cdots, m_{n}$ of $M$, then we shall write simply $N=g s[M]$. A nonsingular matrix $Y(x)$ whose column vectors are solutions of (1) will be called a fundamental matrix for (1). A matrix $M(x)$ will be termed "bounded on $\Delta$ " whenever its individual elements are bounded functions on this interval.

Received by the editors September 6, 1956 and, in revised form, October 12, 1956. 
THEOREM A. If $Y(x)$ is a fundamental matrix for (1), and $T(x)$ $=g s[Y(x)]$, then the corresponding matrix $B(x)$ of $(2)$ is such that:

(i) $B(x)$ is upper-triangular, i.e., $B_{i j} \equiv 0$ for $i>j$;

(ii) if $A(x)$ is bounded on $\Delta$ then $B(x)$ is bounded on $\Delta$;

(iii) the diagonal elements $B_{i i}(x)$ are real-valued.

2. Comments on Theorem A. Perron [3] considered a differential system that in the above notation becomes $\tilde{y}^{\prime}=-\tilde{y} F(x)$. When phrased for the above equation (1) Perron's Theorems 1 and 2 state that if $A(x)$ is bounded on $\Delta$ then there exists a bounded admissible transformation matrix $T(x)$ for which $T^{-1}(x)$ and $T^{\prime}(x)$ are also bounded and such that the matrix $B(x)$ of (2) is upper-triangular and has real-valued diagonal elements; moreover, if $A(x)$ is realvalued then $T(x)$ may be chosen real. In terms of a given fundamental matrix $Y(x)$ Perron defined by certain explicit determinantal formulas a matrix, denoted here by $T(x)$, and then by rather formidable computations showed successively that the corresponding $B(x)$ is upper-triangular, $T(x)$ is unitary, and $T^{\prime}(x)$ is bounded. Although Perron makes no comment on the matter, his explicit formulas constitute a determinantal form of the Gram-Schmidt orthonormalization process. That is, in proving his stated theorems Perron actually established the result of Theorem A, so that this result should be credited to him.

Undoubtedly the failure of Perron to point out the character of the transformation matrix actually occurring in the proofs of his theorems has led to some lack of appreciation of his results. For example, Theorem 1 of Diliberto [1] states that if $A(x)$ is real-valued then there exists an orthogonal admissible transformation matrix $T(x)$ such that the matrix $B(x)$ of (2) is upper-triangular, and that $B(x)$ is bounded whenever $A(x)$ is bounded. Thus for real $A(x)$ this theorem of Diliberto goes beyond the stated Theorems 1 and 2 of Perron [3], whereas, in line with the above remarks, the result actually established by Perron in the proofs of his theorems includes Diliberto's theorem as a special case. It is to be remarked that in terms of a realvalued fundamental matrix Diliberto's transformation matrix, here denoted by $T(x)$, is defined as $g s[Y(x)]$; the upper-triangular character of the associated $B(x)$ is obtained by a relatively simple vector argument, and boundedness of $B(x)$ is deduced from the property that boundedness of $A(x)$ implies boundedness of $T^{\prime}(x)$. As to the proof of this last property Diliberto is incomplete, in that he gives details only of the proof of the boundedness of the first column vector $t_{1}^{\prime}$ of $T^{\prime}$, with no indication as to how one might proceed with the 
remainder of the proof. Actually one may proceed by induction to show that

$$
\begin{aligned}
& t_{1}^{\prime}=A t_{1}-\frac{1}{2} t_{1}\left[t_{1}^{*}\left(A+A^{*}\right) t_{1}\right], \\
& t_{j}^{\prime}=A t_{j}-\sum_{k=1}^{j-1} t_{k}\left[t_{k}^{*}\left(A+A^{*}\right) t_{j}\right]-\frac{1}{2} t_{j}\left[t_{j}^{*}\left(A+A^{*}\right) t_{j}\right], \\
& (j=2, \cdots, n),
\end{aligned}
$$

where it is to be understood that $t_{k}^{*}=\left(t_{k}\right)^{*}$. The details of such proof are rather tedious, although in the opinion of the author it is preferable to the proof of Perron [3] for the corresponding result. Recently, by a simple vector argument Diliberto [2] has given specific formulas for the elements of the matrix of his Theorem 1 corresponding to the matrix $B(x)$ of (2), from which conclusion (ii) is immediate; for the more general case discussed here the formulas corresponding to those of Diliberto are

$$
B_{i i}(x)=\frac{1}{2} t_{i}^{*}\left(A+A^{*}\right) t_{i}, \quad B_{i j}=t_{i}^{*}\left(A+A^{*}\right) t_{j} \text { for } i<j .
$$

It is to be remarked that relations (4) are equivalent to the above equations (3), since $T$ is nonsingular and $T B=A T-T^{\prime}$ by (2).

3. A matrix proof of Theorem A. In the following proof of Theorem A the whole argument is of a matrix character that reduces computational detail to a seeming minimum, while it also highlights two basic properties of admissible transformation matrices that are of interest in themselves. This proof formed part of a paper presented to the American Mathematical Society, (see Reid [4]), although subsequently the paper was not submitted for publication to any journal.

Lemma 1. Let $R$ be a class of $n \times n$ matrices such that; $(\alpha) R$ is closed under multiplication; $(\beta)$ if $M \in R$, and is nonsingular, then $-M^{-1} \in R$; $(\gamma)$ if the elements of $M(x)$ are continuously differentiable on $\Delta$, and $M(x) \in R$ for $x \in \Delta$, then $M^{\prime}(x) \in R$ for $x \in \Delta$. If $Y(x)$ is a fundamental matrix for (1), and $T(x)$ is an admissible transformation matrix such that $Y^{-1}(x) T(x) \in R$ for $x \in \Delta$, then $B(x)$ of (2) is such that $B(x) \in R$ for $x \in \Delta$.

To prove Lemma 1 , one need note only that if $\Gamma=Y^{-1} T \in \mathbb{R}$ for $x \in \Delta$, then $T=Y \Gamma$ and $B=\Gamma^{-1} Y^{-1}\left(-Y \Gamma^{\prime}\right)=-\Gamma^{-1} \Gamma^{\prime} \in R$ for $x \in \Delta$.

LEMMA 2. If $T(x)$ is an admissible transformation matrix, then the 
hermitian matrix $W=T^{*} T$ satisfies with $B(x)$ of (2) the matrix differential equation

$$
W B+B^{*} W+W^{\prime}=T^{*}\left(A+A^{*}\right) T .
$$

Indeed from (2) one has $W B=T^{*} A T-T^{*} T^{\prime}$, from which $B^{*} W$ $=T^{*} A^{*} T-T^{* \prime} T$, and (5) is immediate.

The proof of Theorem A consists in noting that $T(x)=g s[Y(x)]$ is of the form $T(x)=Y(x) \Gamma(x)$, where the elements of $\Gamma(x)$ are continuously differentiable, and for each $x \in \Delta$ the matrix $\Gamma(x)$ belongs to the class $R$ of upper-triangular matrices with real diagonal elements. Such a class $R$ clearly possesses properties $(\alpha),(\beta),(\gamma)$ of Lemma 1, and hence conclusions (i) and (iii) of Theorem A hold. Moreover, as $T(x)$ is unitary (5) of Lemma 2 becomes

$$
B+B^{*}=T^{*}\left(A+A^{*}\right) T
$$

in view of (i), (iii) the matrix equation (6) is equivalent to the formulas (4), and thus conclusion (ii) holds. It is to be emphasized that for real $A(x)$ and real orthogonal $T(x)$ the above matrix derivation of (6) is equivalent to the vector argument of Diliberto [2].

4. Additional remarks. Conclusions (i), (iii) of Theorem $\mathrm{A}$ and the relations (6), or (4), for the elements of the matrix $B(x)$ corresponding to $T(x)=g s[Y(x)]$, together with the fact that if $y, u$ are solutions of (1), (2), respectively, related by $y=T u$ then $|u|=|y|$, imply the following basic result: for any estimate on the growth of the norm of a solution of (1) in terms of bounds for the elements of $A(x)$ there is a corresponding estimate on the growth of the norm of a solution of (1) in terms of bounds for the elements of the hermitian matrix $A(x)+A^{*}(x)$. In particular, if $h(x)$ is such that $\left|\eta^{*}\left[A(x)+A^{*}(x)\right] \zeta\right|$ $\leqq h(x)|\eta||\zeta|$ for arbitrary vectors $\eta, \zeta$, then the matrix $B(x)$ of Theorem A satisfies: $\left|B_{i i}(x)\right| \leqq h(x) / 2 ;\left|B_{i j}(x)\right| \leqq h(x), \quad i<j$; $\left|\eta^{*}\left[B(x)+B^{*}(x)\right] \zeta\right| \leqq h(x)|\eta||\zeta|$ for arbitrary $\eta, \zeta$.

For a general admissible transformation matrix $T(x)$ that is unitary one may show that the $B(x)$ of (2) is such that the real part of the trace of $B(x)$ is equal to the real part of the trace of $A(x)$. This result follows from the argument of Perron [3] used in the proof of his Theorem 3 , or may be established in a manner similar to that employed by Diliberto in the proof of Lemma 4 of [1]. This result is as strong as one may obtain in the indicated direction, for if $\theta_{j}(x),(j=1, \cdots, n)$ are continuously differentiable real-valued functions on $\Delta$, then $T(x)$ $=\left\|\delta_{i j} \exp \left[(-1)^{1 / 2} \theta_{j}(x)\right]\right\|$ is a unitary admissible transformation matrix with the elements of the corresponding matrix $B(x)$ of (2) given by $B_{i j}=A_{i j} \exp \left[(-1)^{1 / 2}\left(\theta_{j}-\theta_{i}\right)\right]-\delta_{i j}(-1)^{1 / 2} \theta_{j}^{\prime}(x)$. 


\title{
BIBLIOGRAPHY
}

1. S. P. Diliberto, On systems of ordinary differential equations, Contributions to the Theory of Nonlinear Oscillations, Annals of Mathematics Studies, no. 20, Princeton, 1950, pp. 1-38.

2. - A note on linear ordinary differential equations, Proc. Amer. Math. Soc. vol. 8 (1957) pp. 462-464. 473.

3. O. Perron, Über eine Matrixtransformation, Math. Zeit. vol. 32 (1930) pp. 465-

4. W. T. Reid, Some results on the growth of solutions of differential systems, Bull. Amer. Math. Soc. Abstract 48-11-319.

NORTHWESTERN UNIVERSITY

\section{AN IRREDUCIBLE UNITARY REPRESENTATION OF A COMPACT GROUP IS FINITE DIMENSIONAL}

\author{
PAUL KOOSIS
}

This note contains a proof of the statement included in the title. The result is certainly known; Anna Hurevitsch [1] proves it using the theorem of Peter and Weyl, under the assumption that the group satisfies the second axiom of countability. It would perhaps nevertheless still be desirable to have at hand a short proof which uses the minimum amount of machinery, then the Peter-Weyl theorem, as well as the rest of the representation theory for compact groups [2, Ch. IV] would follow directly from the classical work of Gelfand and Raikov [3] on the unitary Hilbert space representations of a general locally compact group. Since Cartan and Godement [4] have already shown how the theory of abelian locally compact groups follows from the general one of Gelfand and Raikov, one would have available a rather unified treatment of the two branches of topological group theory which have been most extensively studied, a unity which is not so apparent in existing expositions of the subject (cf. [5]).

Such a proof is given here. It was worked out some time ago, in 1950 , but its publication, put off until now, is maybe yet worth while in view of the above considerations.

(The referee has called my attention to a proof similar to the one given here, save that the condition of complete continuity used below is replaced by that of being an operator of Hilbert-Schmidt type. It

Received by the editors September 5, 1956. 